\title{
Charm Physics at B Factories
}

\author{
Galina Pakhlova \\ Institute for Theoretical and Experimental Physics \\ Moscow - Russia
}

\begin{abstract}
We review of the experimental status in charm mixing, charm semileptonic decays,
\end{abstract} charm baryon spectroscopy and charmonium production at Belle and BaBar.

\section{Charm Mixing}

After the discovery by Tevatron of $B_{s}^{0}-\bar{B}_{s}^{0}$-oscillations $D^{0}$ and $\bar{D}^{0}$ remains the last flavor neutral meson system with unobserved mixing. Neutral meson mixing is characterized by parameters $x=\Delta M / \Gamma$ and $y=\Delta \Gamma / 2 \Gamma$, where $\Delta M$ and $\Delta \Gamma$ are the mass and width difference between the two $C P$ eigenstates. The SM box diagram in $D^{0} \leftrightarrow \bar{D}^{0}$ transitions is strongly GIM and CKM suppressed. However, $D$ mixing can be enhanced by long distance effects, involving on- or off-shell $D^{0} \leftrightarrow \bar{D}^{0}$ transitions through intermediate states accessible to both mesons, that contribute both to $x$ and $y$. New Physics contribution to loops can enhance $x$ only, and observation of $x \gg y$ would be a signal of New Physics. Observation of $\mathrm{CP}$ violating effects in $D$ would be another unambiguous signature of New Physics as the $\mathrm{SM}$ predicts tiny $\mathrm{CP}$ violation beyond the present experimental sensitivity.

Experimentally one of the following techniques is exploited to search for $D$ mixing: study of wrong-sign (WS) hadronic decays, search for WS $D^{0}$ semileptonic decays, a timedependent Dalitz plot analysis or direct measurement of the lifetime difference between opposite $C P$ eigenstates. The first method provides the restrictive mixing constraints, in spite of a complication due to presence of doubly-Cabibbo suppressed (DCS) contribution to the same WS final state. The interference of the mixing and DCS amplitudes results in rotation of measured $x^{\prime}=x \cos \delta+y \sin \delta$ and $y^{\prime}=-x \sin \delta+y \cos \delta$ by the strong phase difference $\delta$ between the mixing and DCS amplitudes. This year BaBar [2] has presented a strong evidence for $D$ mixing in $D^{0} \rightarrow K^{+} \pi^{-}$decays. The WS decay rate is a function of proper decay-time:

$$
R(t)=e^{-\Gamma t}\left(R_{D}+\sqrt{R_{D}} y^{\prime} \Gamma t+\frac{x^{\prime 2}+y^{\prime 2}}{4}(\Gamma t)^{2}\right),
$$

where $R_{D}$ is the DCS decay rate (Fig. 1). The mixing and DCS contributions are thus discriminated in the fit to the time-dependent rate of WS decays. The fit yields the mixing parameters to be $x^{\prime 2}=(-0.22 \pm 0.30 \pm 0.21) \cdot 10^{-3}$ and $y^{\prime}=(9.7 \pm 4.4 \pm 3.3) \cdot 10^{-3}$ and a correlation between them -0.94 . This result is inconsistent with the no-mixing hypothesis with a significance of $3.9 \sigma$. Belle [3] has observed an evidence for $D$ mixing by comparing the apparent lifetime when a $D^{0}$ meson decays to the CP eigenstates $K^{+} K^{-}$and $\pi^{+} \pi^{-}$, and when it decays to the final state $K^{-} \pi^{+}$(Fig. 1). They find $y_{C P}=(1.31 \pm 0.32 \pm 0.25) \%$, $3.2 \sigma$ from zero. Using a time-dependent Dalitz plot analysis Belle [4] has also reported a measurement of $D$ mixing in $D^{0} \rightarrow K_{s}^{0} \pi^{+} \pi^{-}$decays. Assuming negligible CP violation, the mixing parameters were found to be $x=\left(0.80 \pm 0.29_{-0.07-0.14}^{+0.09+0.15}\right) \%$ and $y=(0.33 \pm$ $\left.0.24_{-0.12-0.09}^{+0.07}\right) \%$. Both Belle and BaBar have found no evidence for CP asymmetry in $D$ decays. 

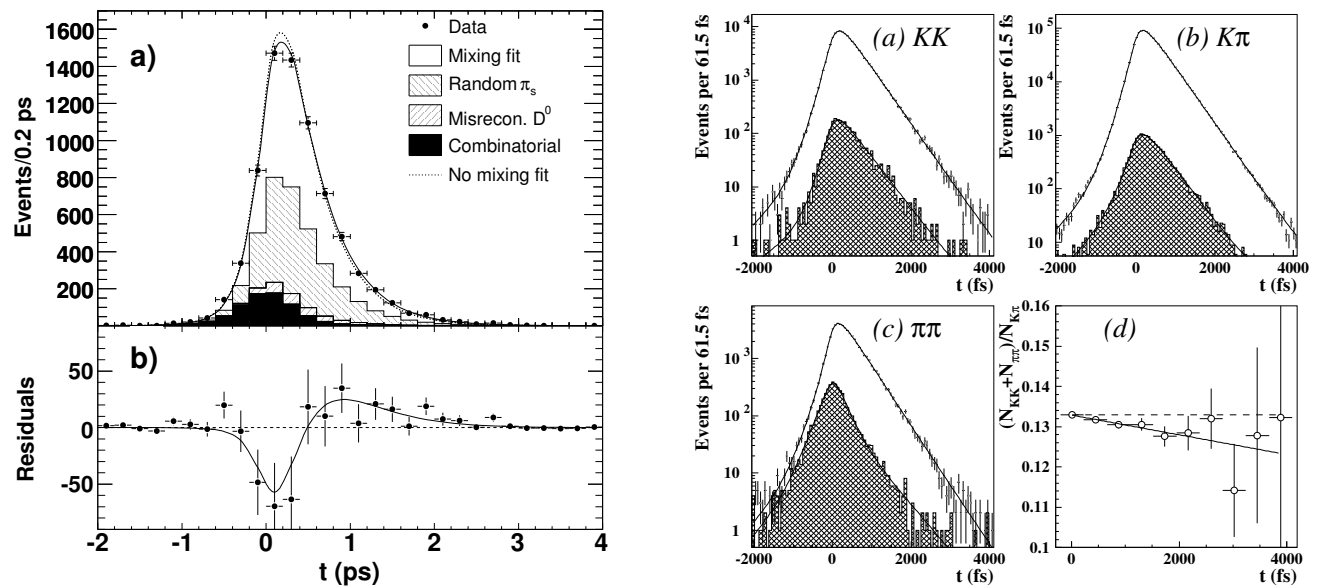

Figure 1: Left plot: (a) BaBar's the proper decay-time distribution of combined $D^{0}$ and $\bar{D}^{0}$ WS candidates; (b) the difference between the data and the no-mixing fit. Right plot: Belle results of the simultaneous fit to decay-time distributions of (a) $D^{0} \rightarrow K^{+} K^{-}$; (b) $D^{0} \rightarrow \pi^{+} \pi^{-}$; (c) $D^{0} \rightarrow K^{-} \pi^{+}$ decays; (d) ratio of decay-time distributions between $D^{0} \rightarrow K^{+} K^{-} / \pi^{+} \pi^{-}$and $D^{0} \rightarrow K^{-} \pi^{+}$.

\section{Charm semileptonic decays}

The measurements of charm semileptonic decay form factors provide a precise tests of LQCD calculations with high statistics and an important inputs for $B$ physics. Belle [5] has measured $D^{0} \rightarrow K^{-} \ell^{+} \nu$ and $D^{0} \rightarrow \pi^{-} \ell^{+} \nu$ decays. The $D^{0}$ momentum was tagged through a full reconstruction of the recoiling charm meson and mesons from fragmentation in the $e^{+} e^{-} \rightarrow c \bar{c}$ events. This technique provides an excellent $q^{2}$ resolution and a low level of backgrounds though with considerably reduced statistics. Normalizing to the total number of $D^{0}$ tags, Belle has measured the absolute branching fractions to be $\mathcal{B}\left(D^{0} \rightarrow K^{-} \ell^{+} \nu\right)=$ $(3.45 \pm 0.07 \pm 0.20) \%$ and $\mathcal{B}\left(D^{0} \rightarrow \pi^{-} \ell^{+} \nu\right)=(0.255 \pm 0.019 \pm 0.016) \%$ and the semileptonic form factors (within the modified pole model) $f_{+}^{K}(0)=0.695 \pm 0.007 \pm 0.022$ and $f_{+}^{\pi}(0)=0.624 \pm 0.020 \pm 0.030$. BaBar [6] has presented a model independent measurements of the hadronic form factor $f_{+}(q 2)$ in the decay $D^{0} \rightarrow K^{-} \ell^{+} \nu$ and the normalization of the form factor at $q^{2}=0$ determined to be $f_{+}(0)=0.727 \pm 0.007 \pm 0.005 \pm 0.007$.

\section{Charm baryon spectroscopy}

Recently the progress in charmed-baryon spectroscopy is evident with a growing number of observed new states and decays modes. BaBar [7] has observed a new charmed baryon in the continuum events in the $D^{0} p$ final state. Fig. 2 shows the $D^{0} p$ invariant mass spectrum with two prominent structures: one near a mass $2880 \mathrm{MeV} / c^{2}$ that is consistent with the known state $\Lambda_{c}(2880)$, the other at a mass of $2939.8 \pm 1.3 \pm 1.0 \mathrm{MeV} / c^{2}$ and with an intrinsic width of $17.5 \pm 5.2 \pm 5.9 \mathrm{MeV}$. As there is no evidence in the $D^{+} p$ spectrum of doubly-charged partners, one can conclude that this state is excited $\Lambda_{c}$, temporary called $\Lambda_{c}(2940)$. Belle [8] reported the first observation of $\Lambda_{c}(2940) \rightarrow \Sigma_{c}(2455) \pi$ decay and measured $\Lambda_{c}(2880)$ and 
$\Lambda_{c}(2940)$ parameters. An analysis of angular distributions in $\Lambda_{c}(2880) \rightarrow \Sigma_{c}(2455) \pi$ decays strongly favors a $\Lambda_{c}(2880)$ spin assignment of $5 / 2$ over $3 / 2$ or $1 / 2$.

Belle [9] has analyzed the $\Lambda_{c}^{+} K^{-} \pi^{+}$final state searching for the doubly-charmed $\Xi_{c c}^{+}(3520)$, reported by SELEX [10]. No evidence for this state is found with the Belle data, while two new charmed strange baryons, $\Xi_{c x}^{+}(2980)$ and $\Xi_{c x}^{+}(3077)$, are clearly seen near the threshold (Fig. 2) with mass of $2978.5 \pm 2.1 \pm 2.0 \mathrm{MeV} / c^{2}\left(3076.7 \pm 0.9 \pm 0.5 \mathrm{MeV} / c^{2}\right)$ and width of $43.5 \pm 7.5 \pm 7.0 \mathrm{MeV}(6.2 \pm 1.2 \pm 0.8 \mathrm{MeV})$, respectively. A significant signal at the mass of $3082.8 \pm 1.8 \pm 1.5 \mathrm{MeV} / c^{2}$ for the isospin partner state decaying into $\Lambda_{c}^{+} K_{S}^{0} \pi^{-}$is also observed. Babar [11] has confirmed observation of $\Xi_{c x}^{+}(2980)$ and $\Xi_{c x}^{+}(3077)$ baryons, with the parameters consistent with the Belle measurement. The high mass of new states suggests that they can be $L=2$ excitations, but no direct measurements of quantum numbers are made so far. Belle [12] has reported a precise measurement of masses of the $\Xi_{c}(2645)$ and
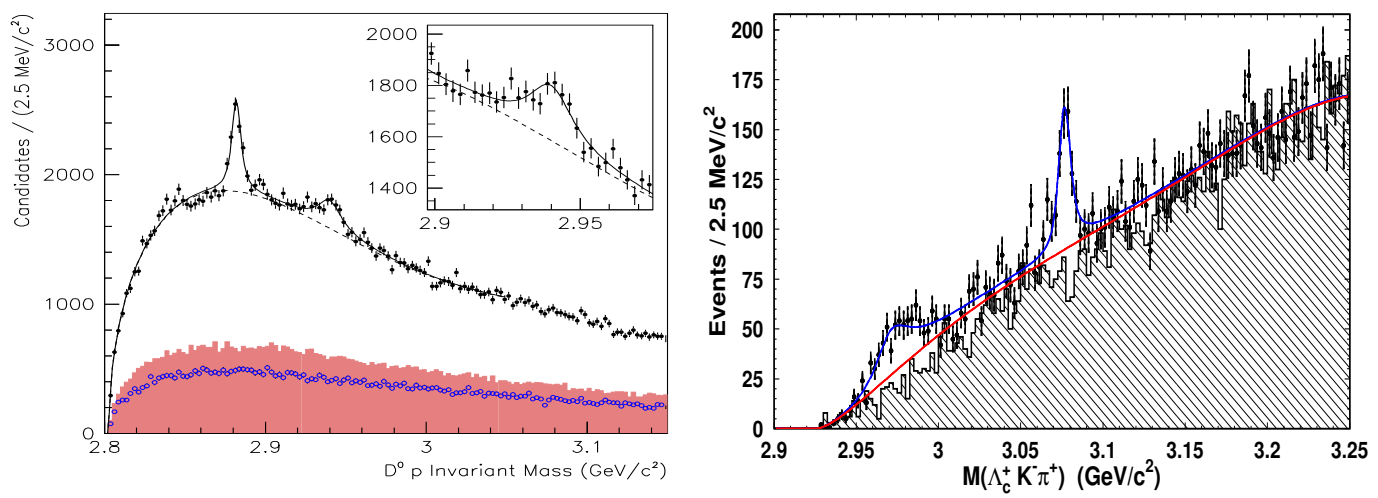

Figure 2: a) The $D^{0} p$ invariant mass distribution and the contribution from false $D^{0}$ candidates estimated from $D^{0}$ mass sidebands and (open points) the mass distribution from $\mathrm{WS} \bar{D}^{0} p$ candidates. b) $\Lambda_{c}^{+} K^{-} \pi^{+}$invariant mass spectrum. The shaded area shows the WS combinations.

$\Xi_{c}(2815)$ baryons. The states $\Xi_{c}(2645)^{0,+}$ are observed in the $\Xi_{c}^{+, 0} \pi^{-,+}$decay modes, while the $\Xi_{c}(2815)^{0,+}$ are reconstructed in the $\Xi_{c}(2645)^{+, 0} \pi^{-,+}$decay modes.

Finally, the family of predicted $J^{P}=3 / 2^{+}$states was completed with the first observation by BaBar [13] of an excited singly-charmed baryon $\Omega_{c}^{*}(c s s)$ in the radiative decay $\Omega_{c}^{0} \gamma$. The mass difference between the $\Omega_{c}^{*}$ and the $\Omega_{c}^{0}$ baryons has been measured to be $70.8 \pm 1.0 \pm$ $1.1 \mathrm{MeV} / c^{2}$ in good agreement with the QCD predictions. From the momentum spectrum of the $\Omega_{c}^{0}$ baryons in the $e^{+} e^{-}$center-of-mass frame $\Omega_{c}^{0}$ production from $B$ decays and in $e^{+} e^{-} \rightarrow c \bar{c}$ events was observed [14].

\section{Measurement of the near-threshold $\sigma\left(e^{+} e^{-} \rightarrow D^{(*)} \bar{D}^{(*)}\right)$ using ISR}

Exclusive $e^{+} e^{-}$hadronic cross sections to final states with charm meson pairs are of special interest because they provide information on the spectrum of $J^{P C}=1^{--}$charmonium states above the open-charm threshold, which is poorly understood. To measure the $e^{+} e^{-}$hadronic cross section at $\sqrt{s}$ smaller than the initial $e^{+} e^{-}$center-of-mass $(\mathrm{CM})$ energy $\left(E_{C M}\right)$ at $B$ factories, initial-state radiation (ISR) can be used. ISR allows a measurement of cross 
sections in a broad energy range while the high luminosity of the $B$-factories compensates for the suppression associated with the emission of a hard photon. BaBar [15] has performed a study of exclusive production of the $D \bar{D}$ system through ISR in a search for charmonium states, where $D=D^{0}$ or $D^{+}$. The $D \bar{D}$ mass spectrum shows a clear $\psi(3770)$ signal. Further structures appear in the 3.9 and $4.1 \mathrm{GeV} / c^{2}$ regions. No evidence is found for $Y(4260)$ decays to $D \bar{D}$, implying an upper limit $\frac{\mathcal{B}(Y(4260) \rightarrow D \bar{D})}{\mathcal{B}\left(Y(4260) \rightarrow J / \psi \pi^{+} \pi^{-}\right)}<7.6(95 \% \mathrm{CL})$. Recently Belle[16] has reported the first measurements of exclusive $e^{+} e^{-} \rightarrow D^{*+} D^{*-}$ and $e^{+} e^{-} \rightarrow D^{+} D^{*-}$ cross sections at $\sqrt{s}$ around the $D^{*+} D^{*-}$ and $D^{+} D^{*-}$ thresholds with ISR. A partial reconstruction technique was used to increase the efficiency and to suppress background. The shape of the $e^{+} e^{-} \rightarrow D^{*+} D^{*-}$ cross section is complicated with several local maxima and minima (Fig. 3). The minimum near $4.25 \mathrm{GeV} / c^{2}$ - in the $Y(4260)$ region - could be due to $D_{s}^{*} D_{s}^{*}\left(D D^{* *}\right)$ threshold effects or due to destructive interference of this state with other $\psi(n S)$ states. Aside from a prominent excess near the $\psi(4040)$, the $e^{+} e^{-} \rightarrow D^{+} D^{*-}$ cross section is relatively featureless. The measured cross sections are compatible within errors

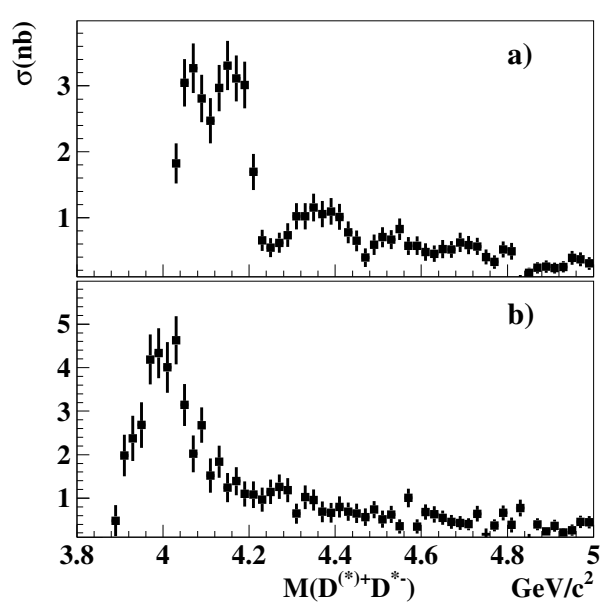

Figure 3: The exclusive cross sections for a) $e^{+} e^{-} \rightarrow D^{*+} D^{*-}$ and b) $e^{+} e^{-} \rightarrow D^{+} D^{*-}$. with the $D^{(*)} \bar{D}^{*}$ exclusive cross section in the energy region up to $4.260 \mathrm{GeV}$ measured by CLEO-c [17].

\section{References}

[1] Slides: http://indico. cern. ch/contributionDisplay . py? contribId=208\&sessionId=5\&conf Id=9499

[2] B. Aubert et al. (BABAR Collab.), Phys. Rev. Lett. 98211802 (2007).

[3] M. Staric et al. (Belle Collab.), Phys. Rev. Lett. 98211803 (2007).

[4] K. Abe et al. (Belle Collab.), arXiv:0704.1000 (2007).

[5] L. Widhalm et al. (Belle Collab.), Phys. Rev. Lett. 97061804 (2006).

[6] B. Aubert et al. (BABAR Collab.), arXiv:0704.0020 (2007).

[7] B. Aubert et al. (BABAR Collab.), Phys. Rev.Lett 98052001 (2007).

[8] K. Abe et al. (Belle Collab.), arXiv:hep-ex/0608043 (2006).

[9] R. Chistov et al. (Belle Collab.), Phys. Rev. Lett.97 162001 (2006).

[10] M. Mattson et al. (SELEX Collab.), Phys. Rev. Lett. 89112001 (2002).

[11] B. Aubert et al. (BABAR Collab.), arXiv:hep-ex/0607042 (2006).

[12] K. Abe et al. (Belle Collab.), arXiv:hep-ex/0608012 (2006).

[13] B. Aubert et al. (BABAR Collab.), Phys. Rev. Lett.97 232001 (2006).

[14] B. Aubert et al. (BABAR Collab.), arXiv:hep-ex/0703030 (2007).

[15] B. Aubert et al. (BABAR Collab.), arXiv:hep-ex/0607083 (2006).

[16] G. Pakhlova et al. (Belle Collab.), Phys. Rev. Lett.98 092001 (2007).

[17] R. Poling (for CLEO Collab.), arXiv:hep-ex/0606016 (2006). 